\title{
StatNet Electroencephalogram: A Fast and Reliable Option to Diagnose Nonconvulsive Status Epilepticus in Emergency Setting
}

\author{
Lady Diana Ladino, Alexandra Voll, Dianne Dash, Wes Sutherland, \\ Lizbeth Hernández-Ronquillo, Jose Francisco Téllez-Zenteno, Farzad Moien-Afshari
}

\begin{abstract}
Background: The StatNet electrode set is a system that can be applied by a non-electroencephalogram (EEG) technologist after minimal training. The primary objectives of this study are to assess the quality and reliability of the StatNet recordings in comparison to the conventional EEG. Methods: Over 10 months, 19 patients with suspected nonconvulsive status epilepticus were included from university hospital emergency settings. Each patient received a StatNet EEG by a trained epilepsy fellow and a conventional EEG by registered technologists. We compared the studies in a blinded fashion, for the timeframe from EEG order to the setup time, start of acquisition, amount of artifact, and detection of abnormalities. The nonparametric Mann-Whitney two-sample t test was used for comparisons. The kappa score was used to assess reliability. Results: Mean age of patients was $61 \pm 16.3$ (25-93) years. The inter-observer agreement for detection of abnormal findings was 0.83 for StatNet and 0.75 for conventional EEG. Nonconvulsive status epilepticus was detected in 10\% (2/19) in both studies. The delay from the time of EEG requisition to acquisition was shorter in the StatNet $(22.4 \pm 2.5$ minutes $)$ than the conventional EEG $(217.7 \pm 44.6$ minutes; $\mathrm{p}<0.0001)$. The setup time was also shorter in the StatNet $(9.9 \pm 0.8$ minutes $)$ compared with the conventional EEG $(17.8 \pm 0.8$ minutes; $\mathrm{p}<0.0001)$. There was no difference in the percentage of artifact duration between the two studies $(\mathrm{p}=0.89)$. Conclusion: This study demonstrates that StatNet EEG is a practical and reliable tool in the emergency setting, which reduces the delay of testing compared with conventional EEG, without significant compromise of study quality.
\end{abstract}

RÉSUMÉ: L'EEG StatNet, une option rapide et fiable pour le diagnostic de crises d'épilepsie non convulsives dans une situation d'urgence. Contexte: Le jeu d'électrodes StatNet est un système qui peut être utilisé par des individus ayant subi un entraînement minimal, qui ne sont pas des techniciens en électroencéphalographie (EEG). Les principaux objectifs de cette étude étaient d'évaluer la qualité et la fiabilité d'enregistrements StatNet par rapport à des enregistrements EEG conventionnels. Méthode: Dix-neuf patients soupçonnés d'être atteints de mal épileptique ont été recrutés dans un contexte d'urgence dans un hôpital universitaire. Ils ont été inclus dans l'étude sur une période de 10 mois. Chaque patient a subi un EEG StatNet effectué par un collègue ayant reçu une formation en épilepsie et un EEG conventionnel effectué par des techniciens diplômés. Nous avons comparé les enregistrements à l'aveugle, quant au délai entre le moment où l'EEG est prescrit et le moment où il est installé, le début de l'enregistrement, la quantité d'artéfacts et la détection d'anomalies. Nous avons utilisé le test non paramétrique de « $\mathrm{t}$ » à deux échantillons de Mann-Whitney pour les comparaisons et le score kappa pour évaluer la fiabilité. Résultats: L'âge moyen des patients était de $61 \pm 16,3$ ans (écart de 25 à 93 ans). La concordance interobservateur pour la détection d'anomalies était de 0,83 pour StatNet et de 0,75 pour l'EEG conventionnel. Un état de mal épileptique non convulsif a été détecté chez $10 \%(2 / 29)$ des patients avec les deux techniques d'enregistrement. Le délai à partir du moment de la réquisition de l'EEG à la réalisation était plus court pour le StatNet (22,4 $\pm 2,5$ minutes) que pour l'EEG conventionnel (217,7 $\pm 44,6$ minutes; $p<0,0001)$. Le temps d'installation était également plus court pour le StatNet $(9,9 \pm 0,8$ minutes) comparé à l'EEG conventionnel $(17,8 \pm 0,8$ minutes; $p<0,0001)$. Nous n'avons pas constaté de différence dans le pourcentage de durée des artéfacts entre les deux techniques $(\mathrm{p}=0,89)$. Conclusion: Cette étude démontre que, dans un contexte d'urgence, l'EEG StatNet est un outil pratique et fiable qui diminue le délai d'exécution du test par rapport à l'EEG conventionnel, sans compromettre significativement la qualité de l'examen.

Keywords: disposable EEG electrodes, emergency department, emergent EEG, intensive care unit, nonconvulsive status epilepticus, stat EEG

doi:10.1017/cjn.2015.391

Can J Neurol Sci. 2016; 43: 254-260

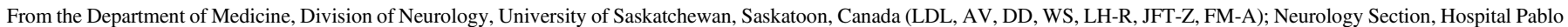
Tobón Uribe, Medellín, Colombia, South America (LDL).

Received May 28, 2015. Final Revisions Submitted November 19, 2015.

Correspondence to: Farzad Moien-Afshari, Division of Neurology, University of Saskatchewan, Royal University Hospital, 103 Hospital Drive, Saskatoon, SK S7N 0W8, Canada,

Email: farzad.moien@usask.ca 


\section{BACKGROUND}

Nonconvulsive status epilepticus (NCSE) is common in critically ill patients ${ }^{1}$ and increases morbidity and mortality. ${ }^{2}$ In patients admitted to intensive care units (ICU) continuous electroencephalogram (EEG) monitoring detected seizure activity in $19 \%$, and almost all episodes were nonconvulsive. ${ }^{3}$ At the emergency department (ED), NCSE was shown to occur in more than $30 \%$ of patients with unexplained alteration of mental status. ${ }^{4}$ Given the subtlety of clinical signs, the diagnosis can be missed without an EEG. ${ }^{5}$

Routine use of hairline EEG as a screening tool for NCSE in the ICU has not been as promising, with sensitivity of $54 \%$ to $72 \%{ }^{7}$ for seizures and $54 \%$ for periodic lateralized epileptiform discharges with the potential to overinterpret benign patterns. ${ }^{7}$ Hence, obtaining an urgent EEG for the diagnosis of NCSE in the ICU and ED is crucial in the management. Unfortunately, it raises logistic difficulties in some hospitals. First of all, the standard procedure for application of electrodes and performing EEG recordings requires substantial training, including complex head measurement for accurate electrode placement, specific skin preparation, and application of conductive paste or glue. ${ }^{8}$ Consequently, a qualified EEG technologist must be available, even if the study is requested after day shift hours. ${ }^{7}$ This entire process takes a considerable amount of time and, in some centers, there are no EEG technologists on call. In fact, many facilities lack immediate and continuous access to EEG testing and its interpretation even during working hours, which directly affects timely treatment of NCSE. Current statistics indicate that the waiting time for a conventional EEG may be several hours to even days, especially on weekends. ${ }^{9,10}$ Furthermore, EEG interpretation by qualified electroencephalographers lags behind the real-time recordings by hours or days, which contribute to further delays in diagnosis and treatment. ${ }^{11}$

Given that NCSE treatment delay is associated with poor outcomes, ${ }^{2}$ performing an EEG as early as possible is important. We present a simplified single-use disposable EEG headpiece system called StatNet (HydroDot, Inc., Westford, MA, USA). This is a peel-and-stick device that can be applied fast with no preparation of the scalp by a non-EEG technologist after minimal training. We compared the quality of the recordings and the ability to detect abnormal findings between the StatNet stat EEG and conventional EEG in the same group of patients. In addition, setup duration and time delay from EEG request to start of acquisition during working hours were compared. We hypothesized that StatNet EEG will be reliable and significantly faster to obtain compared with conventional EEG, without significant compromise of study quality. This method is not intended to replace the current conventional EEG; instead, it supports use after hours when there is no EEG technologist on call. In addition, StatNet can prove very beneficial in remote hospitals where services are not available.

\section{MeTHODS}

Between September 2013 and August 2014, consecutively referred adult patients with clinical suspicion of NCSE from the ED, ICU, or hospital wards were included. This was a prospective cohort study carried out by the Saskatchewan Epilepsy Program in the Royal University Hospital, a tertiary care center in Saskatoon, Saskatchewan, Canada. Patient guardians provided written informed consent. In the majority of cases, it was not possible to obtain consent directly from patients because they were either critically ill for many days, did not have or regain an adequate level of awareness, and, in one case, the patient passed away. The study was approved by Health Canada and the Research Ethics Board at the University of Saskatchewan.

\section{Patient Selection}

Patients with the following criteria were included: 1) adults at 18 years of age or older; 2) any unexplained change or variation in behavior or level of awareness; 3 ) coma or significantly impaired mental status; and 4) one of the following conditions: sepsis, metabolic or toxic abnormalities including drug intoxication with stimulants (cocaine or amphetamines) and lithium, history of epilepsy, witnessed seizures without regaining baseline mental status within 30 minutes, cardiac arrest, clinical features suspicious for status epilepticus such as gaze deviation or nystagmus, mini-myoclonus of fingers, ischemic or hemorrhagic stroke, traumatic brain injury, brain tumor, and central nervous system infection. Neurologists, neurology residents, and emergency physicians received a practical training session for identifying the appropriate patients. They informed the epilepsy fellow during the day shift hours about the patients' clinical history and possible inclusion criteria by a telephone call, without neurologic consultation. They also sent a simultaneous request for a conventional EEG to the Neurophysiology Department. After the call, the epilepsy fellow would attend the patient's bedside as soon as possible to perform the StatNet EEG. Every patient also underwent a routine conventional EEG during the same day.

\section{StatNet and Conventional EEG Setup}

The epilepsy research fellow placed the StatNet electrodes on the patient's head. The fellow received 3 hours of training on how to apply the StatNet EEG electrode set on a dummy's head with hair and how to set up the portable EEG amplifier and the laptop computer for rapid acquisition. The StatNet EEG montage consisted of a longitudinal and transverse bipolar montage without F3, F4, P3, P4, and Pz electrodes. To provide adequate coverage of the parietal region, the lowest posterior electrode line (T5, T6, $\mathrm{O} 1$, and O2) is slightly shifted anteriorly (Figure 1A).

After obtaining informed consent, the patient's largest head circumference $(\mathrm{HC})$ was measured at the level of the nasion and inion to choose the appropriate electrode set size. StatNet electrode sets come in medium and large sizes. Based on manufacturer recommendation, for $\mathrm{HC} \leq 56 \mathrm{~cm}$, a medium set was used; for $\mathrm{HC}$ $>56 \mathrm{~cm}$, a large set was used. The fellow then applied the twopiece designed set (Figure 1B) of disposable single-use electrodes by peeling and sticking it on the patient's scalp. Each electrode was housed under foam padding soaked with conducting gel, thus eliminating the need for cumbersome scalp preparation and application of conducting paste. The silver chloride electrodes are integrated with lead wires, resulting in low ambient noise. The wires were connected to Xltek Trex ambulatory EEG system (Natus Medical Inc, San Carlos, CA, USA), which was then hooked up to a laptop. Although StatNet EEG can provide quality recordings for 4 hours before the conductive gel dries, each recording was conducted for 20 minutes. After the procedure, the epilepsy fellow removed and discarded the electrode set. The EEG traces were potentially uploadable to a central secure EEG server, 
A

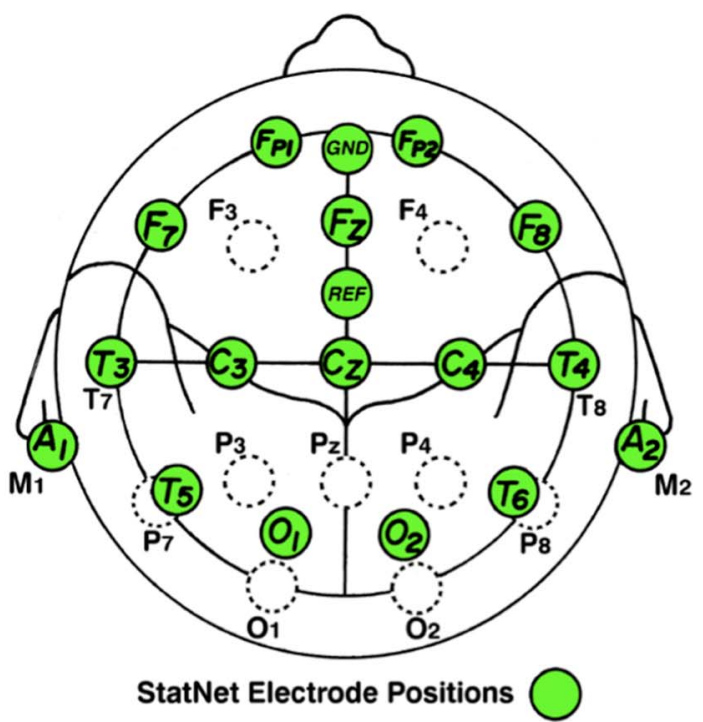

B

A

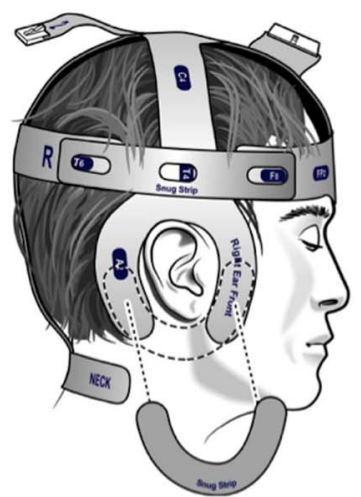

B

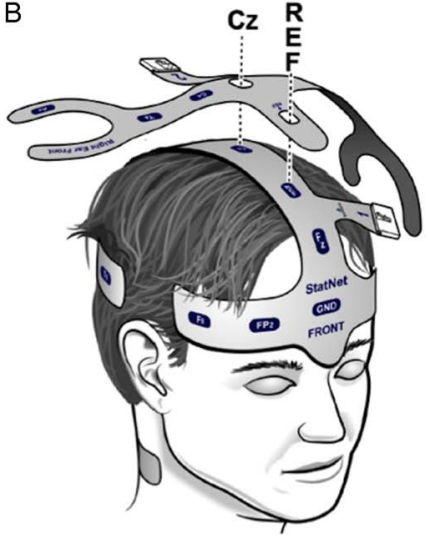

Figure 1: (A) StatNet electrode positions. The StatNet headpiece has fewer electrodes compared with that of a conventional EEG (18 instead of 23); however, it has adequate coverage of all the major areas of the brain. It uses a modified international 10-20 system placement: FP1, FP2, F7, F8, T3, T4, T5, T6, O1, O2, C3, C4, FZ, CZ, A1, A2, REF, and GND. The electrode positions are at approximately $5 \%$ to $10 \%$ of each 10-20 location and are homologous. The posterior electrodes are slightly higher to make it easier to apply to patients in the supine position (www.biosignalgroup.com). Permission has been obtained for use of the submitted figure. (B) Two-piece design StatNet set. The StatNet "peel and stick" EEG disposable headpiece is a pregelled, two-piece, soft-layered integrated circuit connected with a single integrated cable (www.biosignalgroup.com). Permission has been obtained for use of the submitted figure.

which could then be accessed remotely by an on-call electroencephalographer. Results could potentially be communicated via telephone to the requesting physician to guide clinical care. However, for the proof of concept stage, the StatNet EEGs were stored on the recording laptop for future comparison with the conventional EEGs; clinical guidance was provided after the interpretation of the conventional EEG by an electroencephalographer.

Trained and board-certified EEG technologists applied electrodes according to the conventional method for EEG in the same group of patients on the same day. All recordings were carried out on a 32-channel digital EEG acquisition system (Xltek, Natus Medical Inc). The scalp electrodes were placed according to the International 10-20 measurement system using standard gold-plated cup electrodes with conductive paste secured in place with Hypafix and wrapped to conform. Anteroposterior longitudinal bipolar montage was displayed during these digital recordings. Standard activation procedures were employed whenever possible. Each EEG recording was conducted for 30 minutes per Canadian standards. Technologists and the epilepsy fellow documented the setup time in minutes. In addition, the time delay from order to execution of StatNet EEG and the conventional EEG were documented. Chart review of each case was carried out to obtain clinical data including the indication for EEG order and the referring diagnosis. The fellow who performed the StatNet test reassessed the physical examination at the time of the conventional EEG to ensure the clinical possibility of NCSE at the beginning of every test (looking for minimal clinical findings such as nystagmoid eye movements and finger mini-myoclonus).

\section{EEG Interpretation}

Two independent board-certified electroencephalographers (FM-A and JFT-Z) reviewed the conventional EEGs and StatNet EEGs in a blinded fashion. The process of blinding was undertaken by the epilepsy fellow, who converted all EEG montages to the StatNet montage (Figure 2A-D). The tests were randomly coded, and all names, information, and annotations inserted by the technologist were removed. In addition, the duration of all studies was adjusted to the first 20 minutes. The fellow included a short sentence summarizing patients' information including age, sex, and the reason for the study. To keep the studies blinded, the electroencephalographers were not allowed to change the settings or the montage during the reading. The EEG readers were asked to assess each study in four aspects: 1) whether the study was interpretable; 2 ), the presence of artifact in percentage during the recording; and 3) the presence of epileptiform discharges and slowing and, if present, whether they were focal or generalizedif focal, the location, side, and specific patterns such as periodic lateralized epileptiform discharges, frontal intermittent rhythmic delta activity, or temporal intermittent rhythmic delta activity were noted. Triphasic waves and coma patterns (e.g. spindle coma, alpha coma, theta coma, delta coma, burst-suppression) were also identified. Finally, each EEG reader decided whether the EEG was positive for NCSE. The definition of NCSE proposed by Walker et al in 2005 was used. ${ }^{12}$ Electrographic seizure was defined as repetitive generalized or focal spikes, sharp waves, spike-and-slow wave, or sharp-and-slow wave complexes at frequency greater than $3 \mathrm{~Hz}$ or sequential rhythmic, periodic, or quasi-periodic waves at frequency greater than $1 \mathrm{~Hz}$ with definite evolution in frequency, morphology, or location lasting at least 10 seconds. ${ }^{13}$ Their interpretations were then compared with the known diagnosis.

\section{Analysis}

We calculated the agreement between two readers using the kappa coefficient. The interpretation of the kappa values was done according by Landis and Koch. ${ }^{14}$ We compared the time from EEG order request to start of the EEG acquisition and the setup time between both tests (StatNet and conventional) as well as the amount of artifact and ability to detect abnormal findings. 
A

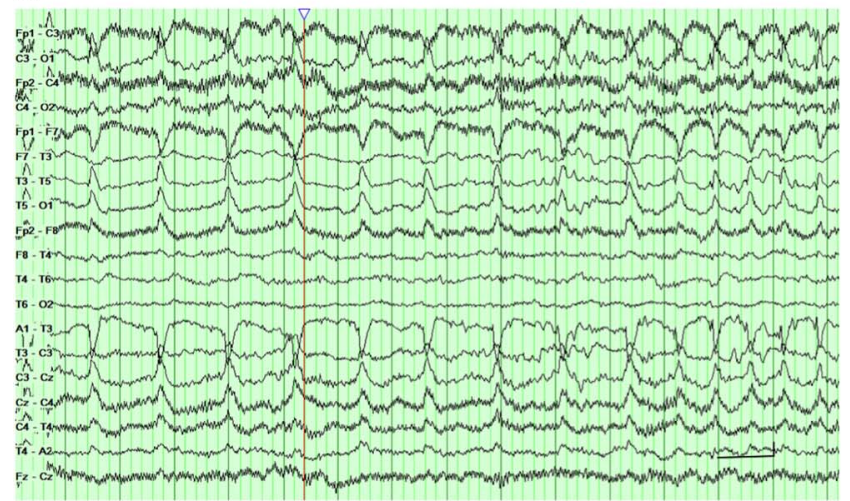

\section{C}

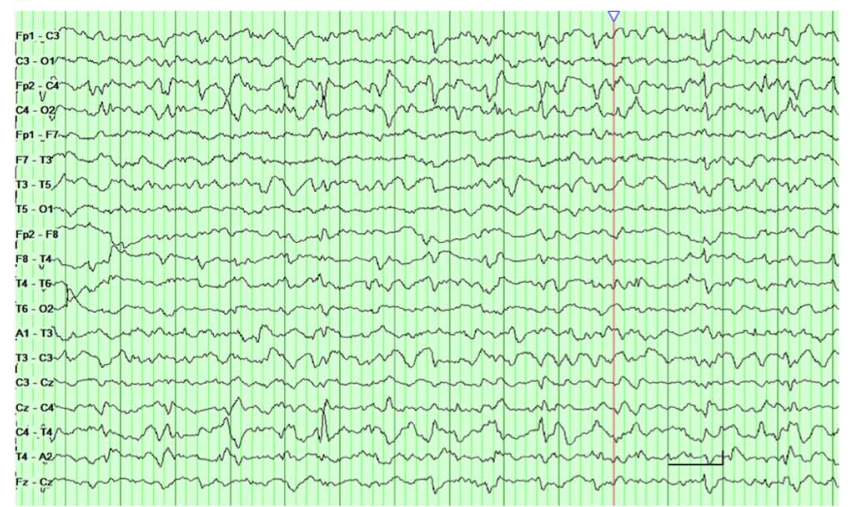

B

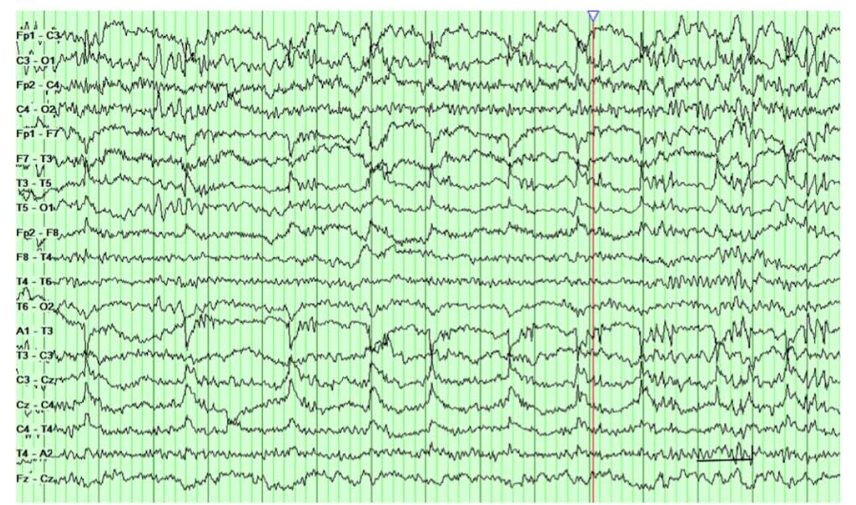

D

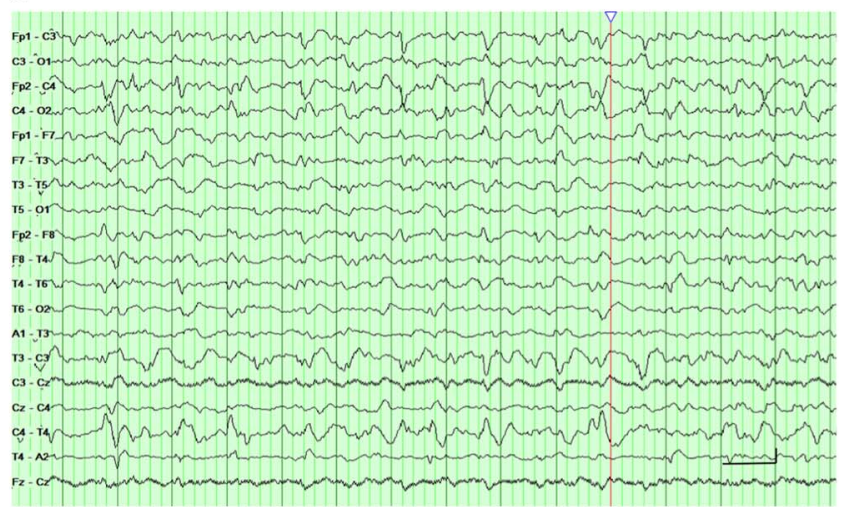

Figure 2: NCSE in two patients. EEG set up on a longitudinal and transverse bipolar montage. Scale represents $1 \mathrm{~s}$ and $50 \mu \mathrm{V}$. (A, B) A continuous left frontotemporal 0.5- to 2-Hz sharp wave activity without clinical manifestations in the patient 1 in a conventional and StatNet $E E G$, respectively. $(C-D)$ A diffuse sharp wave periodic pattern at a frequency of 0.5 to $1 \mathrm{~Hz}$ with maximum activity over the right frontal area in the patient 2 in a conventional and StatNet EEG, respectively.

Nonparametric Mann-Whitney $t$ test was used for comparison. Results are expressed in median minutes \pm standard error of the median. The statistical analysis of the data was performed using commercially available software (SPSS 20; Chicago, IL, USA). The level of significance was set to $\mathrm{p}<0.05$.

\section{RESULTS}

Over 10 months, 19 patients were evaluated. The mean age was $61 \pm 16.3$ ( 25 to 93 ) years and $63 \%$ of the patients were female. The clinical characteristics of the sample are displayed in Table 1 . The majority of the EEG requests came from the ICU and ED; clinical histories were predominantly consistent with an underlying neurological condition (84\%). Non-neurological cases accounted for $16 \%$ of the total: drug intoxication (11\%) and sepsis $(5 \%)$. Six (32\%) patients carried a long-standing epilepsy diagnosis and half of them (16\%) had a history of convulsive status epilepticus. These six patients with known epilepsy diagnosis had witnessed tonic-clonic seizure activity lasting more than 5 minutes, five $(83 \%)$ of them had also witnessed seizure without return of mental status to baseline, and three $(50 \%)$ had positive semiologic findings in the neurological examination suggestive of NCSE. In general, the mean time of mental status depression before the admission was $22 \pm 15$ ( 1 to 48 ) hours. The neurological examination was similar between the two studies.

\section{EEG Validity and Utility}

The inter-observer agreement for the decision of interpretability was almost perfect for StatNet (kappa $=1.0, \mathrm{p}<0.0001)$ and moderate for the conventional group (kappa $=0.76, \mathrm{p}<0.002$ ). The concordance for the detection of abnormal findings was almost perfect for StatNet EEG (0.83) and moderate for conventional EEG $(0.75, p<0.0001)$. The inter-observer agreement in regard to the final diagnosis (normal, encephalopathy, and epileptiform activity) was moderate for StatNet (kappa $=0.7, \mathrm{p}<0.0001)$ and conventional EEG (kappa =0.6, p <0.0001).

There was no difference between the two interpreters in regard to the presence of epileptiform activity. NCSE was detected in $10 \%$ (two of 19 patients) with both studies (Figure 2). Both patients were treated and improved with antiepileptic drugs in the ICU. EEG was normal in $10 \%$ of patients; showed interictal epileptiform discharges that did not evolve into seizures in $10 \%$ (one was a pattern of generalized discharges in a patient with idiopathic epilepsy and suspicion of absence status; the other was a left temporal epileptogenic focus in a patient with a brain tumor); indicated NCSE with periodic discharges in $10 \%$; generalized slowing suggestive of underlying encephalopathy in $60 \%$, and noninterpretable study in $10 \%$, with both StatNet EEG and conventional EEG. There were no patients with clinical seizures during the tests. 
Table 1: General characteristics of the patients

\begin{tabular}{|c|c|}
\hline Category & Total sample $(n=19)$ \\
\hline \multicolumn{2}{|l|}{ Sex } \\
\hline Female & $12(63)$ \\
\hline \multicolumn{2}{|l|}{ Age } \\
\hline Older than 50 years & $16(84)$ \\
\hline \multicolumn{2}{|l|}{ Patient location } \\
\hline $\mathrm{ICU}$ & $9(47)$ \\
\hline ED & $8(42)$ \\
\hline Neurology hospitalization wards & $2(11)$ \\
\hline \multicolumn{2}{|l|}{ Main diagnosis } \\
\hline Epilepsy-NCSE* & $6(32)$ \\
\hline Stroke (ischemic or hemorrhagic) & $6(32)$ \\
\hline CNS infection suspicion & $3(16)$ \\
\hline Drug intoxication & $2(10)$ \\
\hline Brain tumor & $1(5)$ \\
\hline Metabolic disorder & $1(5)$ \\
\hline \multicolumn{2}{|l|}{ Previous epilepsy diagnosis } \\
\hline Symptomatic focal epilepsy & $3(16)$ \\
\hline Idiopathic generalized epilepsy & $3(16)$ \\
\hline Drug resistant epilepsy & $3(16)$ \\
\hline Focal finding in the neurological exam & $6(32)$ \\
\hline
\end{tabular}

$\mathrm{CNS}=$ central nervous system; $\mathrm{ED}=$ emergency department;

ICU $=$ intensive care unit; NCSE = nonconvulsive status epilepticus.

*Patients highly suspected of having NCSE (convulsive seizures followed by unconsciousness)

The patient has a stable intracranial hemorrhage or ischemic stroke but declines from a Glasgow Coma Score of 12 to 7

\section{EEG Timing and Quality}

The mean delay time from EEG order to EEG acquisition was $22.42 \pm 2.53$ minutes for the StatNet group and $217.74 \pm 44.60$ minutes for the conventional group; the difference was significant ( $<<0.0001$, Mann-Whitney $U=23)$ (Figure 3). The mean setup time was $9.98 \pm 0.86$ minutes for the StatNet group and $17.88 \pm 0.81$ minutes for the conventional group; the difference was significant ( $<<0.0001$, Mann Whitney $U=7$ ) (Figure 4).

No difference was noted in the quality of the recordings or the duration of artifacts between the StatNet EEG and the conventional EEG groups. The first EEG reader reported a similar percentage of artifacts in the StatNet and the conventional groups $(61 \pm 37 \%$ vs. $52 \pm 42 \%, \mathrm{p}>0.05)$. The second electroencephalographer reported fewer artifacts in both tests, but there was no difference between the StatNet and the conventional groups $(23 \pm 26$ vs. $27 \pm 34, p>0.05)$. Overall, there was no difference in the percentage of artifacts during the recording between StatNet and conventional EEG, respectively (42\% vs $39 \%, \mathrm{p}>0.05)$.

\section{DISCUSSION}

To the best of our knowledge, there is no prospective study to date that compares the value of StatNet EEG and conventional

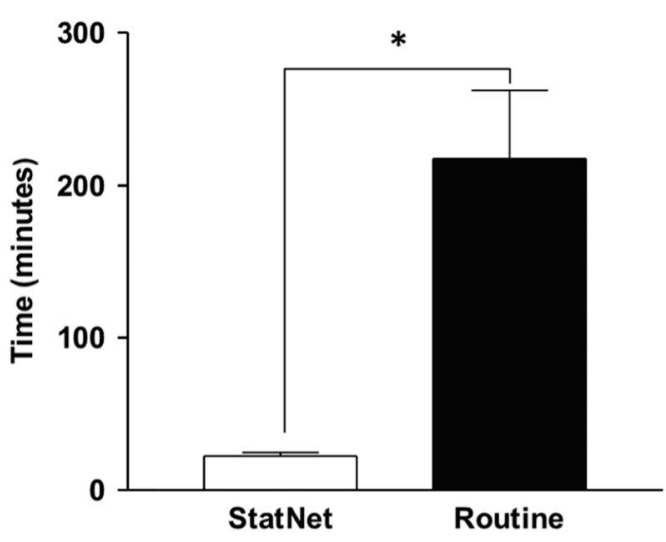

Figure 3: Delay from EEG order to EEG recording.

EEG performed in the same group of patients with suspected NCSE. Our study suggests that StatNet EEG electrode set performs similarly to conventional electrodes in the urgent setting for patients suspected of having NCSE. At the same time, we demonstrated that StatNet EEG has the advantages of being readily available and reliable. In our study, the disposable StatNet EEG electrode set allowed medical staff to fit it to the patient's scalp in less than 10 minutes on average and obtain an acceptable recording.

NCSE can occur after clinically treated convulsive status epilepticus ${ }^{15,16}$; nevertheless, only in $25 \%$ of the cases did noticeable clinical seizures precede EEG diagnosis of NCSE. ${ }^{17}$ Critically ill patients with unexplained alteration of mental status in ICU and ED might also suffer this condition. Epileptic activity was recently found in the EEG of almost half of patients with acute impairment of consciousness, ${ }^{18}$ and it has been demonstrated that delayed diagnosis and treatment of NCSE may lead to increased neurological complications and mortality. ${ }^{16,19}$ There is currently no standardized protocol for identifying the patients with suspected NCSE, and no after-hours urgent EEG availability even in some tertiary care centers. ${ }^{20}$ StatNet EEG could be a solution to this problem, and a more universal use of handy fullmontage quick electrode sets may help lower morbidity and mortality in this population. This could potentially save health care dollars while increasing availability of testing allowing for earlier treatment of NCSE. However, more prospective studies are required to assess this possibility.

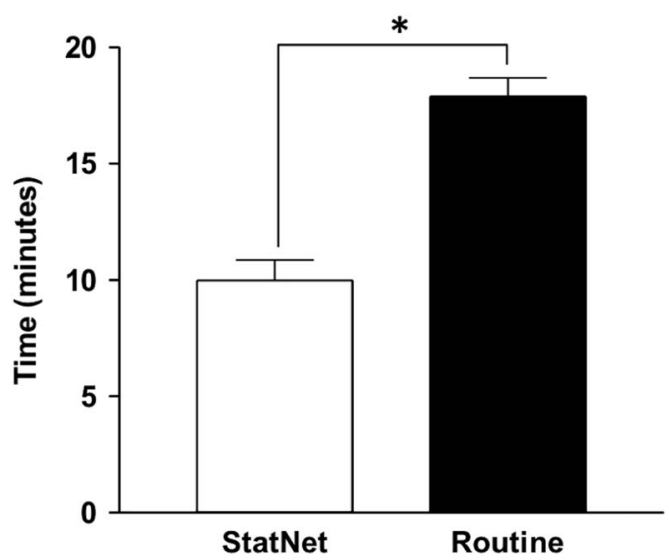

Figure 4: Comparison of setup time between StatNet and conventional EEG 
Our study detected NCSE on stat EEG in $10 \%$ of patients, similar to earlier reports. Varelas et $\mathrm{al}^{21}$ executed a retrospective study in patients with change in mental status or coma. They reported that $11 \%$ of stat EEGs showed NCSE. With similar findings, Alroughani $^{5}$ described a rate of $9 \%$ in patients with unknown cause of impaired level of consciousness, and Bastani et $\mathrm{al}^{22}$ described subclinical epileptiform activity in $12 \%$ of patients studied with a screening EEG device in the ED. More recently, Brenner et $\mathrm{al}^{23}$ found nonconvulsive seizure activity in $8 \%$ of patients when comparing a portable six-lead Brainmaster EEG device with conventional EEG. However, the detection of NCSE in stat EEG varies widely between studies. It has been as low as the study published by Seidel et al, ${ }^{24}$ who found an incidence of $0.8 \%$ among 2514 consecutive patients in a tertiary center, and the series by Lorenzl ${ }^{25}$ and Zehtabachi, ${ }^{26}$ which confirmed NCSE in only $5 \%$ of patients with altered consciousness at a palliative care center and ED, respectively. On the contrary, Privitera and Strawsburg ${ }^{4}$ found NCSE in $34 \%$ of patients who presented with altered consciousness and Praline et $\mathrm{al}^{27}$ reported that $20 \%$ of stat EEG in emergency situations confirmed the presence of NCSE. The low rates of some studies could be due to using only 20 minutes or shorter stat EEGs and not continuous EEGs. It has been well described that electrographic seizures or NCSE in critically ill patients can be better detected using continuous EEG monitoring. In fact, the sensitivity increased to $80 \%-90 \%$ by extending the recording time to $24-48$ hours. ${ }^{3,5,28,29}$ Other bias to take into account is the selection of patients; in more acutely complicated and elderly patients, the possibility of diagnosis is higher. ${ }^{24,26,27}$

As was expected, in our series, StatNet EEG was frequently requested in patients older than age 50 years (84\%) and often located in the ICU and ED $(90 \%)$. Both findings are quite similar to another prospective study done on 111 consecutive stat EEG recordings in which $60 \%$ of patients were between the ages of 50 and 70 years and two-thirds of them came from the ICU and ED, possibly because this group of patients is especially exposed to acute medical problems and neurological disorders. ${ }^{27}$

Finally, the majority of patients in our study $(60 \%)$ showed a diffuse polymorphic slowing. This finding is comparable with earlier reports in which diffuse slowing was seen in half of the ICU-ordered stat EEGs ${ }^{26,30}$ and up to $70 \%{ }^{31}$ of ED patients with alteration of mental status. Although slowing is a nonspecific manifestation of cerebral dysfunction, its magnitude may correlate with the functional severity of clinical encephalopathy.

Each StatNet electrode set costs $\$ 100$. Because StatNet is performed by a physician and not a technologist, it could potentially save costs associated with calling back a technologist after hours. In addition, StatNet EEG may potentially save money by earlier patient discharge from the ED or an overall shorter duration of hospital stay; however, our study was not designed to assess such parameters. In the future, if this device achieves approval from Health Canada, more studies could be designed to look at cost effectiveness of using StatNet EEG in the ED or in remote areas.

\section{Limitations}

This study has a few limitations. The main limitation is the small sample size, although the sample size is reasonable for a pilot study designed to assess feasibility and validity of StatNet EEG compared with conventional EEG. We believe that this objective was accomplished in our study. We did not identify patients who received anticonvulsant medications before the recording or in between StatNet and conventional EEG, which could potentially change the electrical findings. We did not study the clinical benefits of stat EEGs in this study because it was a feasibility study only. The potential therapeutic benefit of StatNet EEG could be assessed in a prospective study. StatNet EEG and conventional EEG were not recorded at the same time and, potentially, some patients in NCSE on one test may not be in NCSE later. Fortunately, in our study, all patients with NCSE were correctly diagnosed with both tests. Finally, it is likely that emergency physicians referred patients with higher pretest probability of NCSE for enrollment and not every patient with alteration of mental status was referred; subjecting the study to a sampling bias. However, this will not affect the comparison between the two studies.

\section{Conclusion}

StatNet EEG is a practical and reliable test when compared with conventional EEG for detection of NCSE. It reduces the delay in testing and treatment in patients suspected of having NCSE, in particular, after hours when there is limited or no access to EEG services. The StatNet method does not replace conventional EEG; however, it can be beneficial by providing earlier assessment. Future studies with larger patient populations may assess the clinical outcomes of patients assessed with StatNet and the use of this device in remote hospital settings.

\section{ACKNOWLEDGMENTS}

We thank the physicians, nurses, and electroencephalogram (EEG) technologists of the Royal University Hospital emergency department and intensive care unit for their cooperation in this study, particularly the EEG technologists who performed the conventional EEGs.

\section{Disclosures}

FM-A has served as a researcher and has received research grants from the College of Medicine of the University of Saskatchewan, the Saskatchewan Health Research Foundation (SHRF), and Ralston Brothers Research Trust. JFT-Z has served as a researcher and has received research grants from the University of Saskatchewan and the Royal University Hospital Foundation. AV, DD, LDL, WS, and LH-R do not have anything to disclose.

\section{REFERENCES}

1. Dunne JW, Summers QA, Stewart-Wynne EG. Non-convulsive status epilepticus: a prospective study in an adult general hospital. Q J Med. 1987;62:117-26.

2. Shneker BF, Fountain NB. Assessment of acute morbidity and mortality in nonconvulsive status epilepticus. Neurology. 2003; 61:1066-73.

3. Claassen J, Mayer SA, Kowalski RG, Emerson RG, Hirsch LJ. Detection of electrographic seizures with continuous EEG monitoring in critically ill patients. Neurology. 2004;62:1743-8.

4. Privitera MD, Strawsburg RH. Electroencephalographic monitoring in the emergency department. Emerg Med Clin North Am. 1994;12:1089-100.

5. Alroughani R, Javidan M, Qasem A, Alotaibi N. Non-convulsive status epilepticus; the rate of occurrence in a general hospital. Seizure. 2009;18:38-42. 
6. Tanner AE, Särkelä MO, Virtanen J, et al. Application of subhairline EEG montage in intensive care unit: comparison with full montage. J Clin Neurophysiol. 2014;31:181-6.

7. Kolls BJ, Husain AM. Assessment of hairline EEG as a screening tool for nonconvulsive status epilepticus. Epilepsia. 2007;48: 959-65.

8. Teplan M. Fundamentals of EEG measurement. Measure Sci Rev. 2002;2:1-11.

9. Ganesan K, Appleton R, Tedman B. EEG departments in Great Britain: a survey of practice. Seizure. 2006;15:307-12.

10. Keenan N, Sadlier LG. Paediatric EEG provision in New Zealand: a survey of practice. N Z Med J. 2015;128:43-50.

11. Trevathan E, Ellen R. Grass Lecture: rapid EEG analysis for intensive care decisions in status epilepticus. Am J Electroneurodiagnostic Technol. 2006;46:4-17.

12. Walker M, Cross H, Smith S, et al. Nonconvulsive status epilepticus: epilepsy research foundation workshop reports. Epileptic Disord. 2005;7:253-96.

13. Chong DJ, Kirsch LJ. Which EEG patterns warrant treatment in the critically ill? Reviewing the evidence for treatment of periodic epileptiform discharges and related patterns. J Clin Neurophysiol. 2005;22:79-91.

14. Landis JR, Koch GG. The measurement of observer agreement for categorical data. Biometrics. 1977;33:159-74.

15. DeLorenzo RJ, Waterhouse EJ, Towne AR, et al. Persistent nonconvulsive status epilepticus after the control of convulsive status epilepticus. Epilepsia. 1998;39:833-40.

16. Drislane FW, Lopez MR, Blum AS, Schomer DL. Detection and treatment of refractory status epilepticus in the intensive care unit. J Clin Neurophysiol. 2008;25:181-6.

17. Laccheo I, Sonmezturk $\mathrm{H}$, Bhatt $\mathrm{AB}$, et al. Non-convulsive status epilepticus and non-convulsive seizures in neurological ICU patients. Neurocrit Care. 2015;22:202-11.

18. Ricardo JA, França MC Jr, Lima FO, Yassuda CL, Cendes F. The impact of EEG in the diagnosis and management of patients with acute impairment of consciousness. Arq Neuropsiquiatr. 2012;70:34-9.

19. Firosh Khan S, Ashalatha R, Thomas SV, Sarma PS. Emergent EEG is helpful in neurology critical care practice. Clin Neurophysiol. 2005;116:2454-9.
20. Abend NS, Dlugos DJ, Hahn CD, Hirsch LJ, Herman ST. Use of EEG monitoring and management of non-convulsive seizures in critically ill patients: a survey of neurologists. Neurocrit Care. 2010;12:382-9.

21. Varelas PN, Spanaki MV, Hacein-Bey L, Hether T, Terranova B. Emergent EEG: indications and diagnostic yield. Neurology. 2003;61:702-4.

22. Bastani A, Young E, Shaqiri B, et al. Screening electroencephalograms are feasible in the emergency department. J Telemed Telecare. 2014;20:259-62.

23. Brenner JM, Kent P, Wojcik SM, Grant W. Rapid diagnosis of nonconvulsive status epilepticus using reduced-lead electroencephalography. West J Emerg Med. 2015;16:442-6.

24. Seidel S, Aull-Watschinger S, Pataraia E. The yield of routine electroencephalography in the detection of incidental nonconvulsive status epilepticus-a prospective study. Clin Neurophysiol. 2012;123:459-62.

25. Lorenzl S, Mayer S, Feddersen B, Jox R, Noachtar S, Borasio GD. Nonconvulsive status epilepticus in palliative care patients. J Pain Symptom Manage. 2010;40:460-5.

26. Zehtabchi S, Abdel Baki SG, Omurtag A, et al. Prevalence of non-convulsive seizure and other electroencephalographic abnormalities in ED patients with altered mental status. Am J Emerg Med. 2013;31:1578-82.

27. Praline J, Grujic J, Corcia P, et al. Emergent EEG in clinical practice. Clin Neurophysiol. 2007;118:2149-55.

28. Claassen J, Jette N, Chum F, et al. Electrographic seizures and periodic discharges after intracerebral hemorrhage. Neurology. 2007;69:1356-65.

29. Narayanan JT, Murthy JM. Nonconvulsive status epilepticus in a neurological intensive care unit: profile in a developing country. Epilepsia. 2007;48:900-6.

30. Varelas PN, Hacein-Bey L, Hether T, Terranova B, Spanaki MV. Emergent electroencephalogram in the intensive care unit: indications and diagnostic yield. Clin EEG Neurosci. 2004; 35:173-80.

31. Lepola P, Myllymaa S, Töyräs J, et al. A Handy EEG Electrode Set for patients suffering from altered mental state. J Clin Monit Comput. 2015;29:697-705. 